\title{
How to start a heuristic? Utilizing lower bounds for solving the quadratic assignment problem
}

\author{
Radomil Matousek $^{a}$, Ladislav Dobrovsky ${ }^{\text {a }}$ and Jakub Kudela ${ }^{a^{*}}$
}

${ }^{a}$ Institute of Automation and Computer Science, Faculty of Mechanical Engineering, Brno University of Technology, Brno, Czech Republic

\begin{tabular}{l}
\hline C H R O N I C L E \\
\hline Article history: \\
Received July 302021 \\
Received in Revised Format \\
September 212021 \\
Accepted December 312021 \\
Available online \\
December, 312021 \\
\hline Keywords: \\
Heuristics \\
Lower bounds \\
Metaheuristics \\
Quadratic assignment problem \\
Starting values
\end{tabular}

\section{A B S T R A C T}

The Quadratic Assignment Problem (QAP) is one of the classical combinatorial optimization problems and is known for its diverse applications. The QAP is an NP-hard optimization problem which attracts the use of heuristic or metaheuristic algorithms that can find quality solutions in an acceptable computation time. On the other hand, there is quite a broad spectrum of mathematical programming techniques that were developed for finding the lower bounds for the QAP. This paper presents a fusion of the two approaches whereby the solutions from the computations of the lower bounds are used as the starting points for a metaheuristic, called $\mathrm{HC} 12$, which is implemented on a GPU CUDA platform. We perform extensive computational experiments that demonstrate that the use of these lower bounding techniques for the construction of the starting points has a significant impact on the quality of the resulting solutions.

\section{Introduction}

The NP-hard quadratic assignment problem (QAP) in its Koopmans and Beckmann form (Koopmans \& Beckmann, 1957), which is notoriously difficult in practice, can be described as follows (Cela, 2013): The problem is structured on a complete directed graph $G=(V, A)$ with $n$ nodes and $n^{2}$ arcs, together with a set of $n$ facilities that have to be assigned to the nodes. The indices $i, j \in V$ correspond to the nodes, the indices $f, g \in N=\{1, \ldots, n\}$ correspond to the facilities, $b_{i, j} \geq 0$ is a given (directed) distance from node $i$ to node $j, a_{f, g} \geq 0$ is a given flow from facility $f$ to facility $g$. By using binary variables $x_{i, f}=1$ if facility $f$ is assigned to node $i$, and 0 otherwise, the QAP can be stated as the following quadratic $0-1$ optimization problem:

$$
\begin{array}{ll}
\min & \sum_{i \in V} \sum_{f \in N} \sum_{j \in V} \sum_{g \in N} a_{f, g} b_{i, j} x_{i f} x_{j, g} \\
& \sum_{i \in V} x_{i, f}=1 \quad \forall f \in N \\
& \sum_{f \in N} x_{i, f}=1 \quad \forall i \in V \\
& x_{i f} \in\{0,1\} \quad \forall i \in V \forall f \in N,
\end{array}
$$

It is quite well known that the constraint matrix, defined by (2)-(3) is totally unimodular, implying that the optimization of any linear objective function over the QAP feasible set is just a relatively easy linear programming problem, known as the linear assignment problem (LAP) (Burkard et al., 2012).

* Corresponding author

E-mail: Jakub.Kudela@vutbr.cz (J. Kudela)

2022 Growing Science Ltd. doi: $10.5267 /$ j.ijiec. 2021.12 .003 
Despite its rather simple definition, QAP is among the most difficult optimization problems that arise in practice - campus planning problem (Dickey \& Hopkins, 1972), backboard wiring problem (Steinberg, 1961), hospital layout problem (Helber et al., 2016), airport gate assignment (Haghani \& Chen, 1998), turbine runner in electricity generation (Laporte \& Mercure, 1988), statistical analysis (Hubert \& Schultz, 1976), and optimal placing of letters on touchscreen devices (Dell'Amico et al., 2009) have all been modeled as a QAP. There are several other well-known combinatorial optimization problems which can be formulated as QAPs with specific coefficient matrices (Cela, 2013) - e.g., the travelling salesman problem, graph partitioning and maximum clique, the linear arrangement problem, and packing problems in graphs, to name a few. An intriguing feature of the QAP is that even for some problems of size $n \leq 50$, such as sko42 or tai30a from the QAPLIB problem library (Burkard et al., 1997), the optimal solution is still not confirmed. Even finding an $\varepsilon$-optimal solution is a difficult problem. There are, however, several QAP instances/structures for which the optimal solution is attainable in polynomial time (Cela et al., 2018) or which were generated in such a way that the optimal solution is known (Li \& Pardalos, 1992). Furthermore, several directions for enriching the QAP formulation have been proposed - among the most notable of these are the multi-objective formulation (Samanta et al., 2018; Sanhueza et al., 2017) and stochastic programming formulation (Popela et al., 2016; Matousek et al., 2017). For these reasons, the study of the QAP attracted quite a large amount of research from both mathematical programming and heuristics communities.

In this paper, we show that the approaches from these two communities can be successfully combined. We will utilize the lower-bounding techniques for the construction of advantageous starting points for a hill climbing metaheuristic and show on extensive computational experiments that starting the heuristic at these points yields significant improvements over the usual random starting points.

The remained of the article is structured as follows: In Section 2, the state-of-the-art in both mathematical programming and heuristics approaches to the QAP is reviewed. In Section 3 a suitable metaheuristic algorithm HC12 is described. Section 4 provides computational comparison of the mathematical programming approaches, and the results obtain from HC12. Conclusions and future research direction are summarized in Section 5.

\section{Methods for approaching QAP}

\section{1. (Meta)Heuristics}

Because of the computational difficulty of the QAP, myriads of heuristics were proposed to tackle this combinatorial problem. Among the first ones were simulated annealing (Burkard \& Rendl, 1984), robust tabu search (Taillard, 1991) and genetic hybrids (Fleurent \& Ferland, 1994) - although these are no longer the most efficient methods, they were able to find the best known solutions for some of the QAPLIB instances, that are yet to be beaten or proven optimal. The comparison between tabu search and simulated annealing based on a size of the QAP was conducted in (Hussin \& Stützle, 2014). A local search heuristic called breakout local search enhanced by a Levenshtein Distance metric for checking solutions for similarity was described in (Aksan et al., 2017). The state-of-the-art in metaheuristics for the QAP includes population based memetic algorithms (Benlic \& Hao, 2015), genetic algorithms (Ahmed, 2015), differential evolution (Hameed et al., 2020) and particle swarm algorithms (Hafiz \& Abdennour, 2016). Hybrid algorithms, combining several heuristics and metaheuristics are also very prevalent. A hybrid teaching-learning based algorithm integrating tabu search within a swarm intelligence metaheuristic was described in (Dokeroglu, 2015). A memetic algorithm that uses a ternary tree structure for its population and the tabu search algorithm, which runs simultaneously, for its local search mechanism was proposed in (Harris et al., 2015). A parallel hybrid algorithm with three phases was proposed by (Tosun, 2015) - this algorithm initially benefits from a genetic algorithm to obtain a high-quality initial seed on which a diversification mechanism is run. Finally, this modified solution is used for a robust tabu search to find a near-optimal result. In (Abdel-Basset et al., 2018) the authors describe an algorithm integrating the whale optimization algorithm with a tabu search. A multistart hyper-heuristic algorithm on the grid is proposed in (Dokeroglu \& Cosar, 2016) - it makes use of different metaheuristics (simulated annealing, robust tabu search, ant colony optimization, and breakout local search) and reports computations on a high-performance cluster with 368 cores and 736 GB of RAM.

Since it offers speed-up opportunity that can outperform current multicore processors, (Tsutsui \& Fujimoto, 2009) applied Graphics Processing Unit (GPU) computation with compute unified device architecture (CUDA) to solve the QAP. In (Czapiński, 2013) is proposed a Parallel Multistart Tabu Search (PMTS) algorithm. It is implemented on a highly powerful GPU hardware intended for high-performance computing with the CUDA platform. Therefore, PMTS is shown to perform competitively with a single-core or a parallel CPU implementation on a high-end six-core CPU. Another GPU based algorithm is described in (Mohammadi et al., 2015) - a parallel genetic algorithm, that (as the authors report) can run up to 30 times faster than its serial counterpart. Finally, the bees algorithm implemented on the CUDA platform is proposed in (Chmiel \& Szwed, 2016).

\subsection{MIP Reformulations}

One common mathematical programming approach for solving the QAP is to "linearize" it, that is, reformulate it as a pure or mixed integer linear programming problem. This was first done in (Gilmore, 1962) by replacing the terms $x_{i, f} x_{j, g}$ in the 
objective function by $n^{4}$ variables $y_{i, f, j, g}=x_{i, f} x_{j, g}$. This reformulation was further improved upon in (Adams \& Johnson, 1994), calling it the level-1 reformulation-linearization technique (RLT-1). The reformulated problem than has the following form:

$$
\begin{array}{ll}
\min & \sum_{i \in V} \sum_{f \in N} \sum_{j \in V} \sum_{g \in N} a_{f, g} b_{i, j} y_{i, f, j, g} \\
& \sum_{i \in V} y_{i, f, j, g}=x_{j, g} \quad \forall j \in V \forall f, g \in N \\
& \sum_{f \in N} y_{i, f, j, g}=x_{j} g \quad \forall i j \in V \forall g \in N \\
& y_{i, f, j, g}=y_{j} g i f \geq 0 \quad \forall i j \in V \forall f g \in N \\
& \sum_{i \in V} x_{i, f}=1 \quad \forall f \in N \\
& \sum_{f \in N} x_{i, f}=1 \quad \forall i \in V \\
& x_{i f} \in\{0,1\} \quad \forall i \in V \forall f \in N,
\end{array}
$$

By relaxing the binary constraint (11) (using a LP relaxation), the above formulation can be used to obtain a valid lower bound on the QAP (1)-(4). The RLT-1 reformulation was further strengthened by introducing additional $n^{6}$ variables, called RLT-2 in (Adams et al., 2007), and even further with additional $n^{8}$ variables, called RLT-3 in (Hahn et al., 2012), which for the time being is still too large even for modern day computers - for problems of size $n=25$, the computations needed to be done on a server with 384 GB of RAM. A different mixed integer linearization scheme, called the Kaufman-Broeckx formulation, was proposed in (Kaufman \& Broeckx, 1978) with $O\left(n^{2}\right)$ additional variables. Although this is the smallest QAP linearization, its LP relaxation is known to be usually weak. This relaxation was tightened in (Xia \& Yuan, 2006) using the Gilmore-Lawler bound (GLB) (Gilmore, 1962; Lawler, 1963) and further enhanced in (Zhang et al., 2013). A formulation based in the Kaufman-Broeckx family was used in (Fischetti et al., 2012) to solve (prove optimality) all the esc instances (Eschermann \& Wunderlich, 1990) (including the one of size $n=128$ ).

\subsection{Lower Bounding Techniques}

Exact solution of a QAP typically requires the use of a branch-and-bound framework (Anstreicher, 2003). In practice, the lack of efficiently computable, tight lower bounds for the QAP has been the key factor in the problem's difficulty, as the tighter the bound is, the more difficult it generally is to compute. There are various approaches for obtaining lower bounds. One of the oldest methods, the Gilmore-Lawler bound (GLB), is still widely used. A comparison of older bounds based on linearization of the QAP can be found in (Karisch et al., 1999). A great success in solving previously unsolved QAP instances was achieved using the convex quadratic programming bound introduced in (Anstreicher \& Brixius, 2001).

A seminal breaking point in combinatorial optimization was the emergence of semidefinite programming (SDP). The SDP bounds for the QAP were first studied in (Zhao et al., 1998). The problem with this relaxation was that it involved a matrix variable of order $n^{2}$, and can therefore only be solved efficiently by interior point methods for, say, $n \leq 20$. This limitation has encouraged research into exploiting group symmetry of the QAP data matrices to obtain smaller and more tractable SDP problems (de Klerk \& Sotirov, 2012). It has also prompted recent research into SDP relaxations of QAP where the matrix variables are of order $n$; see (Peng et al., 2010) and (Peng et al., 2015). In both these lines of research the authors were able to compute the best-known lower bounds for some QAPLIB instances. As we will use the lower bounding techniques for the construction of starting points for our metaheuristic, we will describe these techniques in greater detail. The computation of the GLB can be done in the following way: Denote the row vectors of matrices $A$ and $B$ by $a_{i}$ and $b_{i}, i=1,2, \ldots, n$, Let $\hat{a}_{i}$ be the vector consisting of the $(n-1)$ components of $a_{i}$, without $a_{i, i}$, and let $\hat{b}_{i}$ be the vector consisting of the $(n-1)$ components of $b_{i}$, without $b_{i, i}$. Define a matrix $L=\left(l_{i, j}\right)$ as follows:

$$
l_{i j}=\left\langle\hat{a}_{i}, \hat{b}_{j}\right\rangle_{-}, \quad i, j=1,2, \ldots, n,
$$

where $\langle a, b\rangle_{-}$is the minimal scalar product, which can be computed by ordering the vector $a$ nondecreasingly and $b$ nonincreasingly. The GLB is given by the optimal value of the $n$-dimensional LAP with cost matrix $l_{i, j}+a_{i, i} b_{j, j}$ :

$$
\begin{array}{ll}
\min & \sum_{i=1}^{n} \sum_{j=1}^{n}\left(l_{i, j}+a_{i, i} b_{j, j}\right) x_{i j} \\
& \sum_{i \in V} x_{i, f}=1 \quad \forall f \in N
\end{array}
$$




$$
\begin{aligned}
& \sum_{f \in N} x_{i, f}=1 \quad \forall i \in V \\
& x_{i f} \in\{0,1\} \quad \forall i \in V \quad \forall f \in N
\end{aligned}
$$

which requires only $O\left(n^{3}\right)$ computational time.

The second type of the QAP lower bounds are the eigenvalue bounds. These use the fact that the set of permutation matrices $\Pi_{n}$ can be characterized as:

$$
\Pi_{n}=\mathcal{Q}_{n} \cap \mathcal{E}_{n} \cap \mathcal{N}_{n},
$$

where $\mathcal{Q}_{n}$ is the set of orthogonal matrices, $\mathcal{E}_{n}$ is the set of doubly stochastic matrices and $\mathcal{N}_{n}$ is the set of matrices with positive elements of size $n \times n$.

The QAP can then be equivalently formulated as:

$\min _{X \in \Pi_{n}} \operatorname{tr}\left(A X B X^{T}\right)$,

where $\operatorname{tr}(\cdot)$ is the trace of a matrix. The first eigenvalue bound that uses this QAP formulation was introduced in (Hoffman $\&$ Wielandt, 2003) and is based on the relaxation of the feasible region:

$\min _{X \in Q_{n}} \operatorname{tr}\left(A X B X^{T}\right)=\langle\lambda(A), \lambda(B)\rangle_{-}$,

where $\lambda(\cdot)$ denotes the vector of eigenvalues of the matrix. This bound can be computed with very little effort but tends to be extremely weak. The improvement of this bound was done in (Hadley et al., 1992) and is called the Hadley-RendlWolkowitz (HRW): Let $u_{n}$ be a vector of all ones and let $V$ be an $n \times(n-1)$ matrix with $V^{T} u_{n}=0$ and $\operatorname{rank}(V)=n-$ 1. Then

$$
\left\{X \in \mathcal{R}^{n \times n}: X u_{n}=X^{T} u_{n}=u_{n}\right\}=\left\{\frac{1}{n} u_{n} u_{n}^{T}+V M V^{T}: M \in \mathcal{R}^{(n-1) \times(n-1)}\right\}
$$

which can be used to reparametrize the trace formulation as:

$$
\operatorname{tr}\left(A X B X^{T}\right)=\operatorname{tr}\left(\left(V^{T} A V\right) \hat{X}\left(V^{T} B V\right) \hat{X}^{T}\right)+\frac{2}{n} \operatorname{tr}\left(A J_{n} B\right) X^{T}-\text { const }
$$

where $J_{n}=u_{n} u_{n}^{T}$ is a matrix of all ones, and use the eigenvalue bound to obtain the improved HRW bound:

$$
\left\langle\lambda\left(V^{T} A V\right) \lambda\left(V^{T} B V\right)\right\rangle_{-}+\operatorname{LAP}\left(\frac{2}{n} A J_{n} B\right)-\text { const }
$$

The third type of the QAP lower bounds are based on a convex quadratic programming relaxation of the trace reparameterization shown above. (Anstreicher \& Brixius, 2001) used the above-mentioned parametrization and showed that the following convex quadratic optimization problem gives a lower bound on the QAP:

$\min$

s.t.

where

$$
\begin{aligned}
& \operatorname{vec}(X)^{T} Q \operatorname{vec}(X)+\left\langle\lambda\left(V^{T} A V\right) \lambda\left(V^{T} B V\right)\right\rangle_{-} \\
& X u_{n}=X^{T} u_{n}=u_{n} \quad X \geq 0,
\end{aligned}
$$

$$
Q=(B \otimes A)-\left(I \otimes V \hat{S} V^{T}\right)-\left(V \hat{T} V^{T} \otimes I\right)
$$

and $\hat{S}$ and $\hat{T}$ can be obtained from the spectral decomposition of $V^{T} A V$ and $V^{T} B V$. The last type of the QAP lower bounds we consider are based on a SDP relaxation developed by (Peng et al., 2015): Let $\left(B_{1}, B_{2}\right)$ be a minimal trace matrix splitting of the matrix $B$ and compute a decomposition $B_{i}=\hat{B}_{i}^{T} \hat{B}_{i}$. Let $B_{S}=B_{1}+B_{2}$ the SDP relaxation model of QAP based on minimal trace matrix splitting is the following:

$$
\begin{array}{cl}
\min & \operatorname{tr}(\mathrm{AY}) \\
\text { s.t. } \quad & Y=Y_{1}-Y_{2} \quad Y_{s}=Y_{1}+Y_{2} \\
& \left(\begin{array}{cc}
I & \hat{B}_{1} X^{T} \\
X \hat{B}_{1} & Y_{1}
\end{array}\right) \geq 0\left(\begin{array}{cc}
I & \hat{B}_{2} X^{T} \\
X \hat{B}_{2} & Y_{2}
\end{array}\right) \geq 0 \\
& \operatorname{diag}\left(Y_{1}\right)=X \operatorname{diag}\left(B_{1}\right) \quad Y_{1} e=X B_{1} e \\
& \operatorname{diag}\left(Y_{2}\right)=X \operatorname{diag}\left(B_{2}\right) \quad Y_{2} e=X B_{2} e \\
& \left(X \min \left(\left[B_{1}\right]_{o f f}\right)\right)_{i} \leq\left[Y_{1}\right]_{i j} \quad \forall i \neq j \\
& {\left[Y_{1}\right]_{i j} \leq\left(X \max \left(\left[B_{1}\right]_{o f f}\right)\right)_{i} \quad \forall i \neq j} \\
& \left(X \min \left(\left[B_{2}\right]_{o f f}\right)\right)_{i} \leq\left[Y_{2}\right]_{i j} \quad \forall i \neq j
\end{array}
$$




$$
\begin{aligned}
& {\left[Y_{2}\right]_{i j} \leq\left(X \max \left(\left[B_{2}\right]_{o f f}\right)\right)_{i} \quad \forall i \neq j} \\
& \left(X \min \left([B]_{o f f}\right)\right)_{i} \leq[Y]_{i j} \quad \forall i \neq j \\
& {[Y]_{i j} \leq\left(X \max \left([B]_{o f f}\right)\right)_{i} \quad \forall i \neq j} \\
& \left(X \min \left(\left[B_{s}\right]_{o f f}\right)\right)_{i} \leq\left[Y_{s}\right]_{i j} \quad \forall i \neq j \\
& {\left[Y_{s}\right]_{i j} \leq\left(X \max \left(\left[B_{S}\right]_{o f f}\right)\right)_{i} \quad \forall i \neq j} \\
& \left\|\left[Y_{1}\right]_{i}:\right\|_{2} \leq X\left\|\left[B_{1}\right]_{i}:\right\|_{2} \quad \forall i \\
& \left\|\left[Y_{2}\right]_{i}:\right\|_{2} \leq X\left\|\left[B_{2}\right]_{i}:\right\|_{2} \quad \forall i \\
& \left\|[Y]_{i}:\right\|_{2} \leq X\left\|[B]_{i}:\right\|_{2} \quad \forall i \\
& \left\|\left[Y_{s}\right]_{i}:\right\|_{2} \leq X\left\|\left[B_{s}\right]_{i}:\right\|_{2} \quad \forall i \\
& X \geq 0 \quad X e=X^{T} e=e
\end{aligned}
$$

$B_{\text {off }}$ denotes the matrix consisting of all the off-diagonal elements of $B$, i.e., $B_{\text {off }}=B-$ $\operatorname{diag}\left(b_{11}, b_{22}, \ldots, b_{n n}\right), \max (B)$ (or $\left.\min (B)\right)$ denotes the vector whose $i$-th component is the maximal element (or minimal element) in the $i$-th row (denoted by $B_{i, \text {. }}$ ) of $B$.

In order to obtain a starting point (in our case, a starting permutation) for the upcoming heuristic, we use a projection of the matrix obtained from the lower bounding schemes to the space of permutation matrices: Let $\hat{X}$ be a matrix obtained from the computation of the lower bounds. The "closest" permutation matrix $X$ to $\hat{X}$ can be computed by solving the following problem:

$$
\begin{array}{ll}
\min & \sum_{i=1}^{n} \sum_{j=1}^{n}\left(X_{i, j}-\hat{X}_{i, j}\right)^{2} \\
& \sum_{j=1}^{n} X_{i, j}=1 \sum_{i=1}^{n} X_{i, j}=1 \quad \forall i \forall j \\
\text { s.t. } & X_{i j} \in\{0,1\} \quad \forall i \forall j,
\end{array}
$$

The problem above can be reformulated into an equivalent problem using the fact that $X_{i, j}$ is binary:

which is a simple LAP.

$$
\begin{aligned}
& \sum_{i=1}^{n} \sum_{j=1}^{n}\left(1-2 \hat{X}_{i, j}\right) X_{i, j} \\
& \sum_{j=1}^{n} X_{i, j}=1 \sum_{i=1}^{n} X_{i, j}=1 \quad \forall i \forall j \\
& X_{i j} \in\{0,1\} \quad \forall i \forall j,
\end{aligned}
$$

\subsection{HC12 algorithm}

The binary HC12 algorithm, described in detail in (Matousek \& Zampachova, 2011), is a stochastic heuristic searching algorithm which belongs to the class of pseudo global search methods. The basic step of the algorithm is a generation of a neighborhood of the current solution, which serves as a base for the construction of a new (improved) population. The method of generating the neighborhood is the pivotal characteristic of HC12. The paradigm of the algorithm is the search of the optimal solution in the binary (Hamming) space, that encodes the solution. In this context, it is a parallel approach to genetic algorithms, where the solution is encoded as a binary vector. The best individual of the $i$ th generation (or iteration) is chosen as the base for the following $(i+1)$ generation. The approach is depicted in Fig. 1. The binary vector of the current solution is called a kernel a is denoted with an index "ker" (e.g., $a_{k e r}$ ). The newly generated neighborhood creates a set of $c$ new binary vectors $a_{i}$ with the same length as the vector $a_{k e r}$. These new vectors can be viewed as a population and represented by a matrix $A_{0}=\left(a_{1}, \ldots, a_{c}\right)^{T}$. The degree of locality/globality of the optimization depends on the particular way the new population $A_{0}$ is generated. The goal of the search is to find optimal parameters $x_{\text {opt }}(43)$ with respect to the define objective function $f(x)$ on a parametric space $D \in N$. Because of the binary representation, the parametric space is defined by a mapping $\Gamma:\{0,1\} \rightarrow D$. An important implementation detail of the mapping $\Gamma$ is the translation of the binary vector from the Gray code into direct binary; afterwards, there is a problem-based decoding of the binary vector $(0-1$ 
problem, integer problem, or mixed integer problem). The following relationship is used $x=\Gamma(a)$ to denote the optimal solution as follows:

$$
\begin{aligned}
& x_{\text {opt }}=\operatorname{argmin}_{x \in D} \mathrm{f}(x) \\
& a_{\text {opt }}=\operatorname{argmin}_{a \in\{01\}^{n}} f(\Gamma(a))
\end{aligned}
$$

Over this binary representation is defined the neighborhood relation, that describes the neighborhood $s$ for each feasible $a_{k e r}$ as points $a \in s\left(a_{k e r}\right)$. The choice of the neighborhood function s determines the behavior and character of the HC algorithm (Fig. 1).

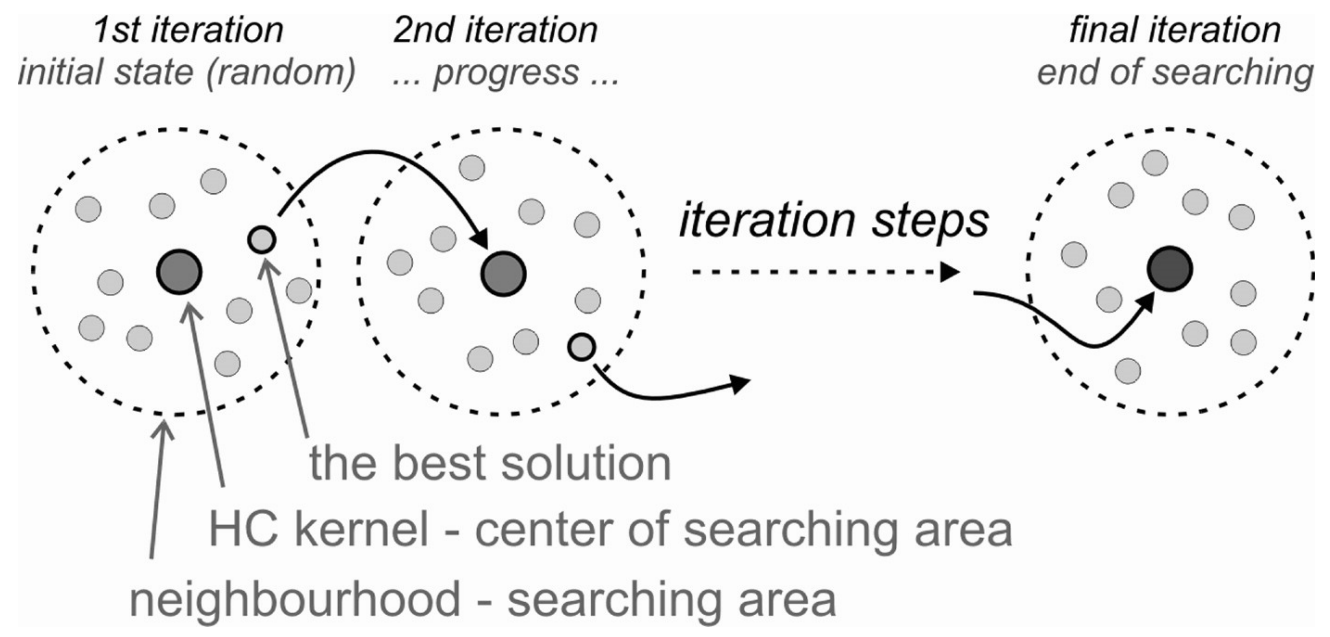

Fig. 1. An schematic example of the progress of the binary $\mathrm{HC}$ algorithm

The HC12 algorithm is very effectively parallelizable. Using the neighborhood function $s$ (45) on a binary vector $a_{k e r}$, the population $A_{0}$ is generated. The set of possible neighborhood functions is denoted by $H(46)$.

$$
\begin{aligned}
& s: a_{\text {ker }} \rightarrow A_{0} \text { i, e, } s:\left\{\begin{array}{lll}
0 & 1
\end{array}\right\}^{n} \rightarrow\left(\left\{\begin{array}{lll}
0 & 1
\end{array}\right\}^{n}\right)^{c} \\
& H=\left\{\begin{array}{llll}
s_{0} & s_{1} & \ldots & s_{n}
\end{array}\right\}
\end{aligned}
$$

The number $c$ of newly generated vectors in the population $A_{0}$ depends on the chosen neighborhood function $S_{k}$ and on the length $n$ of the binary vector $a_{k e r}-$ it is computed as $c=\left(\begin{array}{l}n \\ k\end{array}\right)$. For the realization of the transformations from the set $H$ a system of matrices $M$ is defined. The matrix $M$ corresponding to the function $s_{k}$ will be called a matrix of the $k$-th order and denoted by $M_{k}$. Matrix of the $k$-th order $\left(M_{k}\right)$ is a matrix whose rows represent all points of the Hamming metric space that are distance $k$ from the origin (zero vector of length $n$ ):

$$
\begin{aligned}
& M_{0}=\left(\begin{array}{lllll}
0_{1,1} & 0_{1,2} & \cdots & 0_{1, n}
\end{array}\right) \\
& M_{1}=\left(\begin{array}{ccccc}
1_{1,1} & 0_{1,2} & 0_{1,3} & \cdots & 0_{1, n} \\
0_{2,1} & 1_{2,2} & 0_{2,3} & \cdots & 0_{2, n} \\
\vdots & & & \ddots & \\
0_{c_{1,1}} & 0_{c_{1,2}} & 0_{c_{1,3}} & \cdots & 1_{c_{1}, n}
\end{array}\right) \\
& M_{2}=\left(\begin{array}{ccccc}
1_{1,1} & 1_{1,2} & 0_{1,3} & \cdots & 0_{1, n} \\
1_{2,1} & 0_{2,2} & 1_{2,3} & \cdots & 0_{2, n} \\
\vdots & & & \ddots & \\
0_{c_{2,1}} & 0_{c_{2,2}} & \cdots & 1_{c_{2}, n-1} & 1_{c_{2}, n}
\end{array}\right) \\
& \vdots \\
& M_{n}=\left(\begin{array}{llll}
1_{1,1} & 1_{1,2} & \cdots & 1_{1, n}
\end{array}\right)
\end{aligned}
$$


Using the $k$-th order matrices, the function $s_{k}$ can be effectively computed

as:

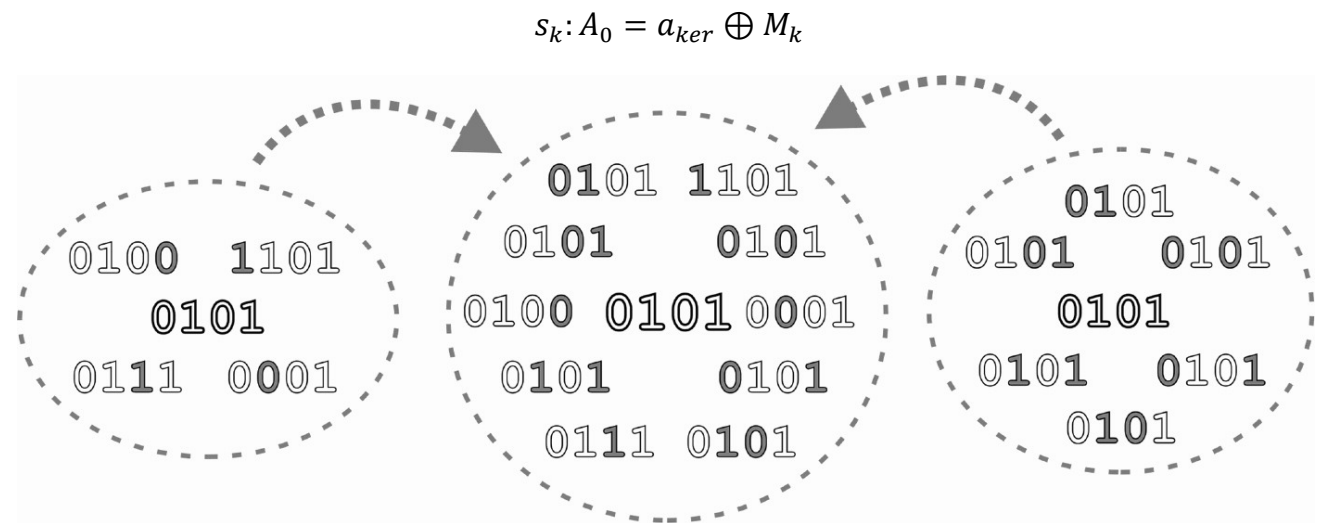

HC1

HC12

$\mathrm{HC2}$

Fig. 2. An example of neighbourhood generation for 4-bit binary string using transformations $H=\left\{s_{0}, s_{1}, s_{2}\right\}$ and matrixes $M_{0}, M_{1}, M_{2}$, i.e. utilization in algorithms $\mathrm{HC} 1, \mathrm{HC} 2$ and their union $\mathrm{HC} 12$

From the practical point of view (because of the combinatorial expansion), only the transformations $M_{0}, M_{1}$, and $M_{2}$ are used. The algorithm HC12 encodes in the last digit of its name the utilized transformations (upto order 2). The general paradigm of the $\mathrm{HC} 12$ algorithm is implemented using several input parameters: fun (indicator of the objective function $f$ ), nRun (the number of runs/restarts of the algorithm). The value $n R u n$ depends on the difficulty of the problem. The section of rows 6 to 10 are the $\mathrm{HC} 12$ algorithm itself. This part is very well (row 8) and well (row 9) parallelizable. The computations in row 8 contain the conversion from the Gray code into direct binary, implicitly. The main focus of this paper is on row 5 of the algorithm. How does one select a good starting solution? One possibility is to start at a random solution with the hope that after a sufficiently large number of tries, one does get a "good enough" solution. The other possibility is to start from a solution that is obtained by some heuristic. In this case, the heuristic in question entails the computation of the lower bound for the QAP (by one of the methods described earlier) and the projection of the obtained lower bound solution on the space of permutation matrices, by solving (40)-(42). The implementation of the HC12 algorithm for the QAP was described in (Matousek et al., 2019). As noted earlier, the optimization problem in (1)-(4) can be interpreted as a search over the space of permutation matrices $X \in \Pi_{n}$. From the problem structure of the QAP it is clear that swapping arbitrary columns of a (feasible) matrix $X$ always results in a different feasible matrix (and swapping the rows of the matrix has the same effect).

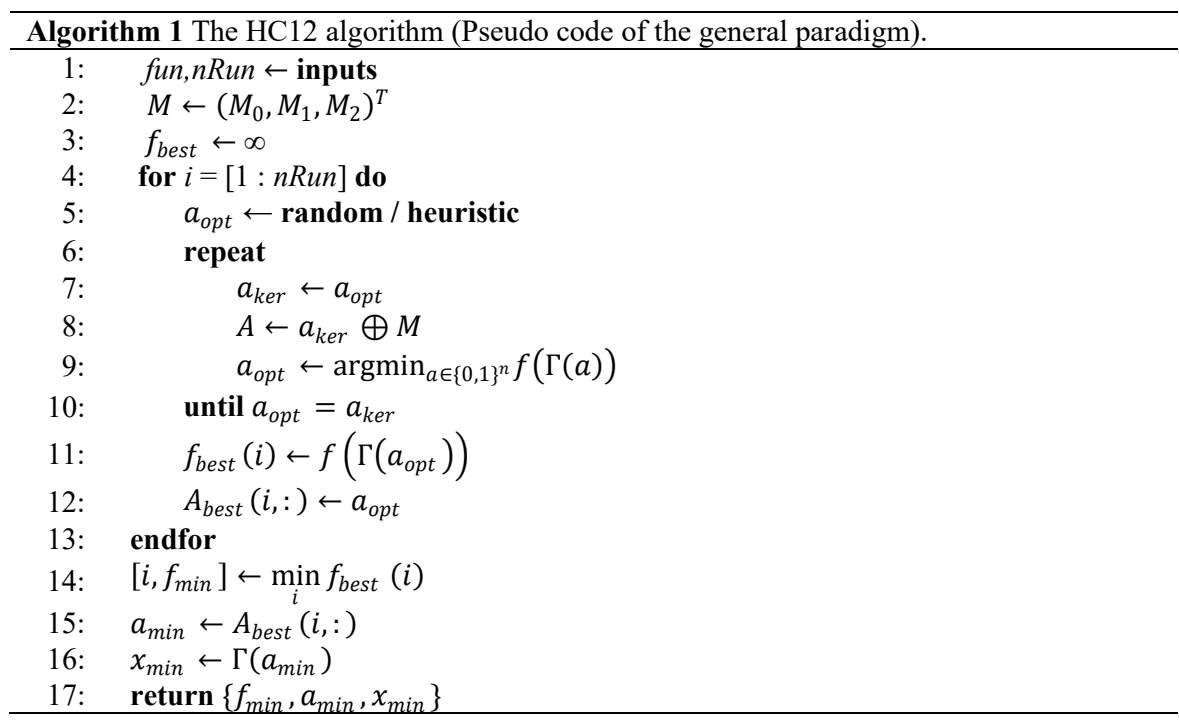




\section{Results and discussion}

The computational experiments were carried out on 53 symmetrical QAP instances from the QAPLIB. For these instances the GLB (12)-(15), HRW (16), convex quadratic (17)-(18), and semidefinite (19)-(36) bounds, and their projections (40)(42) were computed. For the computation of the convex quadratic (AB) and semidefinite (PE) bounds, and for the computation of the LAP for the projection, the corresponding optimization problems were implemented in JuMP environment in JULIA language and the MOSEK solver was used to obtain the solutions. The results from these computations are summarized in Table 1. For one of the instances (tho150), the AB and PE formulations were too big to handle. These computations were carried out on Intel Xeon E5530 2.40GHz CPU with 16GB of RAM. The HC12 algorithm was implemented for HPC computations on GPU CUDA 7.x (i.e., NVIDIA RTX 2080, 8GB), where not more than 6GB were used for any of the QAP instances. From Table 1 we can see that, at least in general, the more complicated formulations (convex quadratic and semidefinite) produce better (higher) lower bounds, but not necessarily better (lower) starting point values, when judged solely on the resulting projection. The trade-off is that these more complicated formulations need quite a lot more computational resources (judged by the computational time) and are only feasible for instances up to $n=100$. Also, every method produced the best lower bound and best projected value for at least one problem instance. It should be noted that the lower bounds are not only useful for constructing possible starting solutions for heuristics, but also help to judge the closeness of the solution obtained by the heuristic to the true optimum. This is especially important in situation, where the is otherwise no information about what the optimal value of the QAP instance might be. Next, we used the projected values from the lower bounding techniques as the starting points for the HC12 metaheuristic and run it 1,000 times for each problem instance. The best results of these simulations (the solution with the lowest objective value out of the 1,000) are reported in Table 2. We also include the results from simulations that used random permutations as the starting point (again 1,000). Similarly, to the results of the lower bounds, there is not a clear winner, as for each of the methods (even for the random start) there are instances where it produced solutions that were better than the ones from the other methods. However, we can compare each of the bounding methods with the random start to see if there is significant difference. This comparison is summarized in Table 3 - we can see that even the "worst" performing lower bounding technique (HWB) was significantly better that random start, beating it in 30 of the 53 instances. The "best" performing lower bounding technique was the most complicated semidefinite formulation (PE), which was better than random start in 41 of the 52 instances. We can also see that the GLB method performed a bit better than the much more complicated convex quadratic $(\mathrm{AB})$ one. Similar pattern can be observed for the median results reported in Table 4 . The main difference is that the random start was never the best scoring method, while each of the lower bounding methods were the best in at least 6 instances. The comparison of the lower bounding method with random start for median results summarized in Table 5 shows even bigger difference than the one for best (minimum) results - the "worst" lower bounding technique (HWB, again) was better than random start in 41 of the 53 instances, and the "best" one (PE, again) was better in every one of the 52 instances. The GLB and AB methods perform similarly well. From these results, it is clear that starting a heuristic from a carefully chosen points leads to an increase in quality of the resulting solutions. The choice of the technique for constructing these starting points mainly depends on the computational resources at our disposal. While for the QAP the semidefinite (PE) formulation produced the best behaving starting points, it was also the most computationally demanding method, requiring the use of advanced convex optimization algorithms or the use of powerful solvers. In contrast to this, the GLB method produce starting points that are almost as good as the PE one, but the computational requirements for GLB are negligible.

\section{Conclusion}

In this paper we have studied the effects of using the lower bounding techniques for the QAP as for the generation of starting points for the HC12 heuristic, that subsequently tried to find the optimal solution for the QAP. We have shown through extensive numerical computations that this utilization of the lower bounding techniques significantly improves the values of the resulting solutions. Out of the four compared lower bounding techniques, the best overall results were obtained by using the semidefinite relaxation method, which was also the most computationally demanding one. When the computational resources, or the access to high quality semidefinite optimization solvers are limited, the GLB bound can serve as an excellent surrogate - although the resulting solutions are not as good, the computational requirements are negligible.

Future research will focus on extending the multicriteria and stochastic QAP instances. Also, the evaluation of various other heuristics that can use the starting points could be interesting, as different methods could benefit more (or less) from starting from an already decent point. Lastly, we expect to work on the evaluation of the starting solutions for other NP-hard optimization problems. 
Table 1

Lower bounds (LB), values of the projections (PV), and computational time (T) of the four considered QAP lower bounding techniques for selected QAPLIB problems (BKW - best known value). Best results (highest for LB and lowest for PV) are emphasized in bold.

\begin{tabular}{|c|c|c|c|c|c|c|c|c|c|c|c|c|c|}
\hline \multirow[b]{2}{*}{ Instance } & \multirow[b]{2}{*}{ BKW } & \multicolumn{3}{|c|}{$\begin{array}{l}\text { GLB } \\
\text { (Gilmore, 1962) }\end{array}$} & \multicolumn{3}{|c|}{$\begin{array}{l}\text { HRW } \\
\text { (Hadley et al., 1992) }\end{array}$} & \multicolumn{3}{|c|}{$\begin{array}{l}\text { AB } \\
\text { (Anstreicher } \\
2001)\end{array}$} & \multicolumn{3}{|c|}{$\begin{array}{l}\text { PE } \\
\text { (Peng et al., 2015) }\end{array}$} \\
\hline & & LB & PV & $\mathrm{T}[\mathrm{s}]$ & LB & $\mathrm{PV}$ & $\mathrm{T}[\mathrm{s}]$ & LB & PV & $\mathrm{T}[\mathrm{s}]$ & LB & PV & $\mathrm{T}[\mathrm{s}]$ \\
\hline chr12a & 9552 & 724 & 44232 & 0.001 & 0 & 25638 & 0.001 & 0 & 23246 & 0.095 & 8499 & 25752 & 1.136 \\
\hline chr12b & 9742 & 7146 & 25580 & 0.001 & 0 & 26712 & 0.001 & 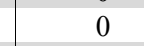 & 27088 & 0.107 & 7340 & 41170 & 1.317 \\
\hline $\operatorname{chr} 12 \mathrm{c}$ & 11156 & 7976 & 18784 & .001 & 0 & 31608 & 0.001 & 0 & 30876 & 0.089 & 9832 & 438 & \\
\hline $\operatorname{chr} 15 a$ & 9896 & 5625 & 50174 & 0.001 & 0 & 25958 & 0.001 & 0 & 25880 & 0.253 & 7441 & 46976 & 2.515 \\
\hline chr15b & 990 & 4653 & 54254 & .001 & 0 & 38470 & 0.001 & 0 & 19008 & 0.256 & 5166 & 30798 & .870 \\
\hline $\operatorname{chr} 15 \mathrm{c}$ & 504 & 165 & 44602 & 0.001 & 0 & 26144 & 0.001 & 0 & 35936 & 0.260 & & & 2.207 \\
\hline chr18a & 11098 & 6779 & 89486 & 0.001 & 0 & 38778 & 0.001 & 0 & 51800 & 0.331 & & & \\
\hline chr18b & 534 & 1534 & 4640 & 0.001 & 0 & 2372 & & 0 & & & & & \\
\hline chr2 & 92 & 2150 & 9778 & 0 & 0 & & & & 6904 & & & & \\
\hline & 2298 & 196 & 10430 & & 0 & & & & 7386 & 0.497 & & & \\
\hline chr20c & 14142 & 8601 & 79430 & 0.013 & 0 & 63268 & 0.0 & 0 & 63350 & 0.511 & & & \\
\hline $\operatorname{chr} 22 \mathrm{a}$ & 6156 & 5924 & 17622 & 0.003 & 0 & 10178 & 0.001 & 0 & 10588 & 0.815 & & & \\
\hline $\mathrm{chr}$ & 94 & 5936 & 13486 & 0.002 & 0 & 9530 & 0. & 0 & 11744 & 0.995 & & & \\
\hline chr25a & 96 & 765 & 18964 & 0.002 & 0 & 14186 & 0. & 0 & 13062 & 1.444 & & 11708 & \\
\hline had20 & 22 & 166 & 7550 & 0.001 & 6625 & & 0 & 71 & 7184 & 0.519 & & & \\
\hline kra30a & 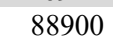 & 68360 & 120000 & 0.0 & 6717 & & 0. & & 111 & & & & \\
\hline & & & & & & & & & 40 & & & & \\
\hline kra32 & & & & & & & & & & & & & \\
\hline nug18 & & 54 & & & & & & & & 0.303 & & & \\
\hline nug20 & 70 & 057 & 80 & 0002 & 2196 & 2940 & 0. & 53 & 2884 & 0.481 & & 72 & \\
\hline nug21 & 38 & 833 & & 00 & 1979 & 2914 & & & 3030 & 27 & & & 5.777 \\
\hline nug22 & & 183 & & 0 & 2 & & & & 4346 & & & & \\
\hline nug24 & & 576 & 42 & 0. & 2 & 70 & & & 4272 & & & & \\
\hline nug25 & & 369 & & 0.0 & & & & & & & & & \\
\hline nug27 & & & & & & & & & & & & & \\
\hline nug & & & & & & & & & & & & & \\
\hline nug30 & & & & & & & & & & & & & \\
\hline scr 15 & 40 & 737 & 54 & 0.0 & & 50 & & & 77786 & 0.299 & & & \\
\hline scr 20 & 0 & 766 & & & & & & & & 0.474 & & & \\
\hline sko42 & & 311 & & & & & & & & 18.23 & & & \\
\hline sko64 & & 522 & 57316 & 0.0 & & & & & 56214 & 126.2 & & & 623.7 \\
\hline sko72 & & & & & & & & & & 222.4 & & & \\
\hline sko81 & & & & & & & & & & 466.3 & & & \\
\hline sko & & & & & & & & & & & & & \\
\hline & & & & & & & & & & & & & \\
\hline & & & & & & & & & & 3055 & & & 10745 \\
\hline sko100c & 52 & 79 & 88 & 0.1 & 11 & 142 & 0. & & & 3306 & & & 920 \\
\hline sko100d & & & 172024 & & & & & & 169774 & 2928 & & 171152 & 10162 \\
\hline sko100e & 9150 & & 171360 & 0.093 & 136996 & 168732 & & 711 & 168640 & 3090 & & 169914 & 9688 \\
\hline sko100f & & & & & 136860 & 170008 & & 138661 & 169090 & 3634 & & 564 & 10627 \\
\hline ste 3 & & & & & 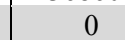 & & & 0 & & 9.257 & & & \\
\hline & & & & & 0 & & & 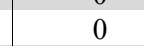 & & & & & \\
\hline & & & & & 0 & & & 0 & & & & & \\
\hline tai25a & $67 \mathrm{e} 6$ & 2417 & $1.457 \mathrm{e} 6$ & 0.002 & 6657 & $1.310 \mathrm{e} 6$ & 0.0 & 207 & $1.366 \mathrm{e} 6$ & 1.758 & & $1 \mathrm{e} 6$ & 12.89 \\
\hline tai50a & $386 \mathrm{e}$ & $54 \mathrm{e} 6$ & $5.838 \mathrm{e} 6$ & 0.017 & $40 \mathrm{e} 6$ & $80 \mathrm{e} 6$ & 0.002 & $70 \mathrm{e} 6$ & $5.613 \mathrm{e} 6$ & 67.49 & $42 \mathrm{e} 6$ & $5.667 \mathrm{e} 6$ & 253.0 \\
\hline tai60a & $205 \mathrm{e} 6$ & & $8.481 \mathrm{e} 6$ & & $5.537 \mathrm{e} 6$ & $8.398 \mathrm{e} 6$ & 0.004 & $5.575 \mathrm{e} 6$ & $8.464 \mathrm{e} 6$ & 82.67 & $.544 \mathrm{e} 6$ & $8.216 \mathrm{e} 6$ & 841.2 \\
\hline tai80a & $49 \mathrm{e} 7$ & $32 \mathrm{e} 7$ & $1.570 \mathrm{e} 7$ & 0.065 & $1.030 \mathrm{e} 7$ & $1.568 \mathrm{e} 7$ & 0.005 & $1.035 \mathrm{e} 7$ & $1.573 \mathrm{e} 7$ & 413.5 & $.031 \mathrm{e} 7$ & $1.556 \mathrm{e} 7$ & 3213 \\
\hline tai100a & $0=7$ & $82 \mathrm{e} 7$ & $2.403 \mathrm{e} 7$ & 0.101 & $1.579 \mathrm{e} 7$ & $2.348 \mathrm{e} 7$ & 0.0 & $1.585 e^{7}$ & $2.347 \mathrm{e} 7$ & 1667 & $1.552 \mathrm{e} 7$ & $2.347 \mathrm{e} 7$ & 6460 \\
\hline tho 30 & 1 & & 195698 & 0.0 & (2) & & & & & 4.067 & & 198154 & 27.20 \\
\hline tho & & & 298906 & & & & & 661 & 319004 & 13.45 & 210 & 3114 & 109.9 \\
\hline tho 150 & $8.133 \mathrm{e} 6$ & $.123 \mathrm{e} 6$ & $9.703 \mathrm{e} 6$ & 0.226 & $7.350 \mathrm{e} 6$ & $9.756 \mathrm{e} 6$ & & - & - & - & - & - & - \\
\hline wil50 & 48816 & 38069 & 53942 & 0.014 & & & 0.003 & & 52938 & 40.64 & & & 281.4 \\
\hline willo0 & 273038 & 210949 & 293908 & 0.095 & 260827 & 293206 & 0.007 & 262584 & 291630 & 2591 & 264724 & 294948 & 11078 \\
\hline
\end{tabular}


Table 2

Best (minimum) results from the simulations. If the BKW is confirmed optimal, it is highlighted in bold. Also, in bold is the method that produced the best solution for the given instance.

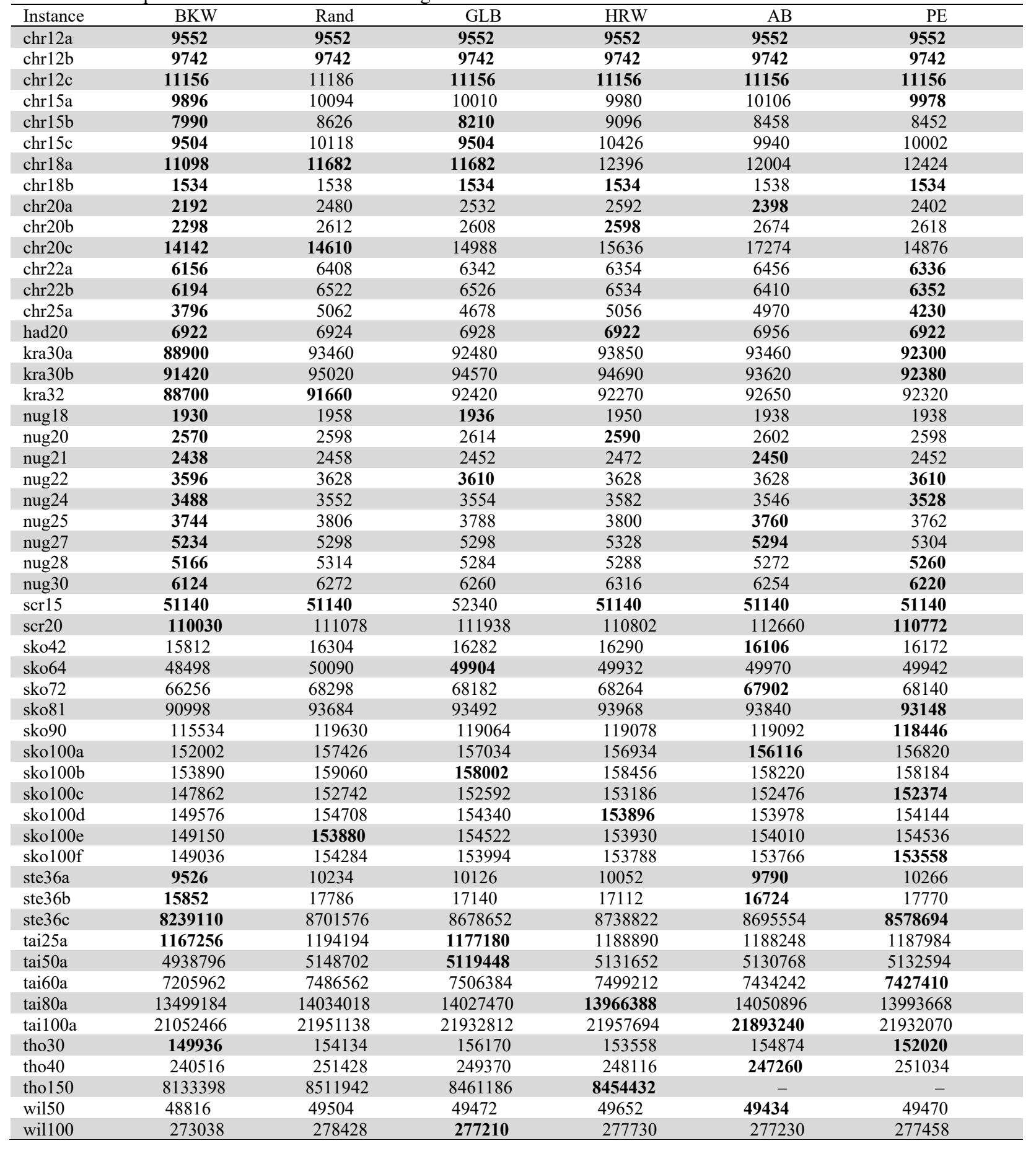

Table 3

Comparison of the lower bounding methods with Rand - best (minimum) results.

\begin{tabular}{lllll}
\hline & GLB & HWB & AB & PE \\
\hline Rand better & 12 & 19 & 13 & 7 \\
Rand worst & 37 & 30 & 33 & 41 \\
Rand equal & 4 & 4 & 6 & 4 \\
\hline
\end{tabular}


Table 4

Median results from the simulations. If the BKW is confirmed optimal, it is highlighted in bold. Also, in bold is the method that produced the lowest median value for the given instance.

\begin{tabular}{|c|c|c|c|c|c|c|}
\hline Instance & BKW & Rand & GLB & HRW & $\mathrm{AB}$ & $\mathrm{PE}$ \\
\hline $\operatorname{chr} 12 a$ & 9552 & 12531 & 11866 & 11550 & 12798 & 12130 \\
\hline $\operatorname{chr} 12 b$ & 9742 & 13170 & 10570 & 11628 & 11548 & 11886 \\
\hline $\operatorname{chr} 12 \mathrm{c}$ & 11156 & 14221 & 13060 & 14139 & 13518 & 13355 \\
\hline chr15a & 9896 & 14691 & 13886 & 13850 & 13625 & 14264 \\
\hline chr15b & 7990 & 13505 & 12334 & 13637 & 12490 & 12142 \\
\hline $\operatorname{chr} 15 \mathrm{c}$ & 9504 & 15445 & 14303 & 15812 & 14518 & 14675 \\
\hline $\operatorname{chr18a}$ & 11098 & 19074 & 18159 & 18664 & 17565 & 17237 \\
\hline chr18b & 1534 & 1779 & 1730 & 1760 & 1776 & 1734 \\
\hline chr20a & 2192 & 3480 & 3374 & 3406 & 3280 & 3359 \\
\hline chr20b & 2298 & 3455 & 3384 & 3468 & 3453 & 3272 \\
\hline chr20c & 14142 & 25957 & 23068 & 24678 & 27073 & 23338 \\
\hline $\operatorname{chr} 22 \mathrm{a}$ & 6156 & 7168 & 7007 & 7016 & 7144 & 6962 \\
\hline chr22b & 6194 & 7218 & 7086 & 7227 & 7078 & 7059 \\
\hline chr25a & 3796 & 6819 & 6068 & 6677 & 6520 & 5687 \\
\hline had20 & 6922 & 7008 & 7002 & 7018 & 6982 & 7000 \\
\hline kra30a & 88900 & 99285 & 98410 & 100070 & 97930 & 97340 \\
\hline kra30b & 91420 & 100360 & 100945 & 100275 & 100695 & 100145 \\
\hline kra32 & 88700 & 98365 & 98360 & 98435 & 98340 & 98260 \\
\hline nug18 & 1930 & 2038 & 2022 & 2010 & 2018 & 2012 \\
\hline nug20 & 2570 & 2728 & 2708 & 2708 & 2704 & 2714 \\
\hline nug21 & 2438 & 2596 & 2562 & 2570 & 2548 & 2560 \\
\hline nug22 & 3596 & 3789 & 3738 & 3878 & 3740 & 3734 \\
\hline nug24 & 3488 & 3754 & 3731 & 3744 & 3689 & 3670 \\
\hline nug25 & 3744 & 4002 & 3950 & 3930 & 3920 & 3940 \\
\hline nug27 & 5234 & 5588 & 5544 & 5532 & 5497 & 5486 \\
\hline nug28 & 5166 & 5546 & 5492 & 5492 & 5462 & 5460 \\
\hline nug30 & 6124 & 6562 & 6490 & 6524 & 6518 & 6474 \\
\hline scr15 & 51140 & 57428 & 56850 & 56240 & 56604 & 56613 \\
\hline scr20 & 110030 & 125945 & 124260 & 122999 & 121845 & 122096 \\
\hline sko42 & 15812 & 16854 & 16728 & 16830 & 16661 & 16663 \\
\hline sko64 & 48498 & 51228 & 50996 & 51092 & 50894 & 50853 \\
\hline sko 72 & 66256 & 69782 & 69362 & 69625 & 69278 & 69267 \\
\hline sko81 & 90998 & 95591 & 95186 & 95379 & 95093 & 94751 \\
\hline sko90 & 115534 & 121745 & 121200 & 120730 & 121122 & 120980 \\
\hline sko100a & 152002 & 159834 & 159447 & 159059 & 158770 & 159425 \\
\hline sko100b & 153890 & 161739 & 160704 & 160686 & 160100 & 160450 \\
\hline sko100c & 147862 & 156070 & 155364 & 155557 & 154906 & 155008 \\
\hline sko100d & 149576 & 157353 & 156497 & 156886 & 156012 & 156377 \\
\hline sko100e & 149150 & 157467 & 156798 & 156325 & 156646 & 156178 \\
\hline sko100f & 149036 & 156510 & 155907 & 155665 & 155841 & 155841 \\
\hline ste36a & 9526 & 11375 & 11134 & 11126 & 11062 & 11192 \\
\hline ste $36 b$ & 15852 & 21794 & 20978 & 20601 & 20783 & 20899 \\
\hline ste $36 \mathrm{c}$ & 8239110 & 9523806 & 9564108 & 9602558 & 9566412 & 9411506 \\
\hline tai25a & 1167256 & 1227125 & 1223279 & 1226160 & 1223803 & 1226389 \\
\hline tai50a & 4938796 & 5266951 & 5249145 & 5255063 & 5235616 & 5234108 \\
\hline tai60a & 7205962 & 7629342 & 7632746 & 7622290 & 7617218 & 7592056 \\
\hline tai80a & 13499184 & 14235086 & 14239312 & 14251040 & 14249678 & 14214487 \\
\hline tai100a & 21052466 & 22265769 & 22209784 & 22277072 & 22202771 & 22230955 \\
\hline tho30 & 149936 & 162522 & 163144 & 162024 & 161934 & 159821 \\
\hline tho40 & 240516 & 262386 & 259343 & 259822 & 258655 & 262188 \\
\hline tho 150 & 8133398 & 8647360 & 8609647 & 8594449 & - & - \\
\hline wil50 & 48816 & 50434 & 50269 & 50544 & 50092 & 50126 \\
\hline willo0 & 273038 & 280670 & 280037 & 279836 & 279480 & 279705 \\
\hline
\end{tabular}

Table 5

Comparison of the lower bounding methods with Rand - median results

\begin{tabular}{lllll}
\hline & GLB & HWB & AB & PE \\
\hline Rand better & 5 & 12 & 5 & 0 \\
Rand worst & 47 & 41 & 47 & 52 \\
\hline
\end{tabular}


Acknowledgement

This work was supported by IGA BUT: FSI-S-20-6538.

\section{References}

Abdel-Basset, M., Manogaran, G., El-Shahat, D., \& Mirjalili, S. (2018). Integrating the whale algorithm with Tabu search for quadratic assignment problem: A new approach for locating hospital departments. Applied soft computing, 73, 530-546.

Adams, W. P., \& Johnson, T. A. (1994). Improved linear programming-based lower bounds for the quadratic assignment problem. DIMACS series in discrete mathematics and theoretical computer science, 16, 43-77.

Adams, W. P., Guignard, M., Hahn, P. M., \& Hightower, W. L. (2007). A level-2 reformulation-linearization technique bound for the quadratic assignment problem. European Journal of Operational Research, 180(3), 983-996.

Ahmed, Z. H. (2015). A multi-parent genetic algorithm for the quadratic assignment problem. Opsearch, 52(4), 714-732.

Aksan, Y., Dokeroglu, T., \& Cosar, A. (2017). A stagnation-aware cooperative parallel breakout local search algorithm for the quadratic assignment problem. Computers \& Industrial Engineering, 103, 105-115.

Anstreicher, K. M., \& Brixius, N. W. (2001). A new bound for the quadratic assignment problem based on convex quadratic programming. Mathematical Programming, 89(3), 341-357.

Anstreicher, K. M. (2003). Recent advances in the solution of quadratic assignment problems. Mathematical Programming, 97(1), 27-42.

Benlic, U., \& Hao, J. K. (2015). Memetic search for the quadratic assignment problem. Expert Systems with Applications, 42(1), 584-595.

Burkard, R. E., \& Rendl, F. (1984). A thermodynamically motivated simulation procedure for combinatorial optimization problems. European Journal of Operational Research, 17(2), 169-174.

Burkard, R. E., Karisch, S. E., \& Rendl, F. (1997). QAPLIB-a quadratic assignment problem library. Journal of Global optimization, 10(4), 391-403.

Burkard, R. E., Dell'Amico, M., \& Martello, S. (2012). Assignment problems: revised reprint. Society for Industrial and Applied Mathematics.

Cela, E. (2013). The quadratic assignment problem: theory and algorithms (Vol. 1). Springer Science \& Business Media.

Cela, E., Deineko, V., \& Woeginger, G. J. (2018). New special cases of the Quadratic Assignment Problem with diagonally structured coefficient matrices. European journal of operational research, 267(3), 818-834.

Chmiel, W., \& Szwed, P. (2016). Bees algorithm for the quadratic assignment problem on CUDA platform. In Man-Machine Interactions 4 (pp. 615-625). Springer, Cham.

Czapiński, M. (2013). An effective parallel multistart tabu search for quadratic assignment problem on CUDA platform. Journal of Parallel and Distributed Computing, 73(11), 1461-1468.

de Klerk, E., \& Sotirov, R. (2012). Improved semidefinite programming bounds for quadratic assignment problems with suitable symmetry. Mathematical programming, 133(1), 75-91.

Dell'Amico, M., Diaz, J. C. D., Iori, M., \& Montanari, R. (2009). The single-finger keyboard layout problem. Computers \& Operations Research, 36(11), 3002-3012.

Dickey, J. W., \& Hopkins, J. W. (1972). Campus building arrangement using TOPAZ. Transportation Research, 6(1), 59-68.

Dokeroglu, T. (2015). Hybrid teaching-learning-based optimization algorithms for the quadratic assignment problem. Computers \& Industrial Engineering, 85, 86-101.

Dokeroglu, T., \& Cosar, A. (2016). A novel multistart hyper-heuristic algorithm on the grid for the quadratic assignment problem. Engineering Applications of Artificial Intelligence, 52, 10-25.

Eschermann, B., \& Wunderlich, H. J. (1990). Optimized synthesis of self-testable finite state machines. In 20th international symposium on fault-tolerant computing (FTCS 20).

Fischetti, M., Monaci, M., \& Salvagnin, D. (2012). Three ideas for the quadratic assignment problem. Operations research, 60(4), 954-964.

Fleurent, C., \& Ferland, J. A. (1994). Genetic hybrids for the quadratic assignment problem. Quadratic assignment and related problems, 16, 173-187.

Gilmore, P. C. (1962). Optimal and suboptimal algorithms for the quadratic assignment problem. Journal of the society for industrial and applied mathematics, 10(2), 305-313.

Hadley, S. W., Rendl, F., \& Wolkowicz, H. (1992). A new lower bound via projection for the quadratic assignment problem. Mathematics of Operations Research, 17(3), 727-739.

Hafiz, F., \& Abdennour, A. (2016). Particle Swarm Algorithm variants for the Quadratic Assignment Problems-A probabilistic learning approach. Expert Systems with Applications, 44, 413-431.

Haghani, A., \& Chen, M. C. (1998). Optimizing gate assignments at airport terminals. Transportation Research Part A: Policy and Practice, 32(6), 437-454.

Hahn, P. M., Zhu, Y. R., Guignard, M., Hightower, W. L., \& Saltzman, M. J. (2012). A level-3 reformulation-linearization technique-based bound for the quadratic assignment problem. INFORMS Journal on Computing, 24(2), 202-209.

Hameed, A., Aboobaider, B., Mutar, M., \& Choon, N. (2020). A new hybrid approach based on discrete differential evolution algorithm to enhancement solutions of quadratic assignment problem. International Journal of Industrial Engineering Computations, 11(1), 51-72. 
Harris, M., Berretta, R., Inostroza-Ponta, M., \& Moscato, P. (2015, May). A memetic algorithm for the quadratic assignment problem with parallel local search. In 2015 IEEE congress on evolutionary computation (CEC) (pp. 838-845). IEEE.

Helber, S., Böhme, D., Oucherif, F., Lagershausen, S., \& Kasper, S. (2016). A hierarchical facility layout planning approach for large and complex hospitals. Flexible services and manufacturing journal, 28(1), 5-29.

Hoffman, A. J., \& Wielandt, H. W. (2003). The variation of the spectrum of a normal matrix. In Selected Papers Of Alan J Hoffman: With Commentary (pp. 118-120).

Hubert, L., \& Schultz, J. (1976). Quadratic assignment as a general data analysis strategy. British journal of mathematical and statistical psychology, 29(2), 190-241.

Hussin, M. S., \& Stützle, T. (2014). Tabu search vs. simulated annealing as a function of the size of quadratic assignment problem instances. Computers \& operations research, 43, 286-291.

Kaufman, L., \& Broeckx, F. (1978). An algorithm for the quadratic assignment problem using Bender's decomposition. European Journal of Operational Research, 2(3), 207-211.

Karisch, S. E., Cela, E., Clausen, J., \& Espersen, T. (1999). A dual framework for lower bounds of the quadratic assignment problem based on linearization. Computing, 63(4), 351-403.

Koopmans, T. C., \& Beckmann, M. (1957). Assignment problems and the location of economic activities. Econometrica: journal of the Econometric Society, 53-76.

Matousek, R., \& Zampachova, E. (2011). Promising GAHC and HC12 algorithms in global optimization tasks. Optimization methods and software, 26(3), 405-419.

Matousek, R., Popela, P., \& Kudela, J. (2017). Heuristic approaches to stochastic quadratic assignment problem: Var and cvar cases. Mendel, 23(1), 73-78.

Matousek, R., Dobrovsky, L., \& Kudela, J. (2019, July). The quadratic assignment problem: metaheuristic optimization using HC12 algorithm. In Proceedings of the Genetic and Evolutionary Computation Conference Companion (pp. 153-154).

Mohammadi, J., Mirzaie, K., \& Derhami, V. (2015, November). Parallel genetic algorithm based on GPU for solving quadratic assignment problem. In 2015 2nd International Conference on Knowledge-Based Engineering and Innovation (KBEI) (pp. 569-572). IEEE.

Laporte, G., \& Mercure, H. (1988). Balancing hydraulic turbine runners: A quadratic assignment problem. European Journal of Operational Research, 35(3), 378-381.

Lawler, E. L. (1963). The quadratic assignment problem. Management science, 9(4), 586-599.

Li, Y., \& Pardalos, P. M. (1992). Generating quadratic assignment test problems with known optimal permutations. Computational Optimization and Applications, 1(2), 163-184.

Peng, J., Mittelmann, H., \& Li, X. (2010). A new relaxation framework for quadratic assignment problems based on matrix splitting. Mathematical Programming Computation, 2(1), 59-77.

Peng, J., Zhu, T., Luo, H., \& Toh, K. C. (2015). Semi-definite programming relaxation of quadratic assignment problems based on nonredundant matrix splitting. Computational Optimization and Applications, 60(1), 171-198.

Popela, P., Matousek, R., \& Kudela, J. (2016). Heuristic approaches to stochastic quadratic assignment problem: VO and MM cases. Mendel 22(1), 117-122.

Samanta, S., Philip, D., \& Chakraborty, S. (2018). Bi-objective dependent location quadratic assignment problem: Formulation and solution using a modified artificial bee colony algorithm. Computers \& Industrial Engineering, 121, 826.

Sanhueza, C., Jiménez, F., Berretta, R., \& Moscato, P. (2017, June). PasMoQAP: a parallel asynchronous memetic algorithm for solving the multi-objective quadratic assignment problem. In 2017 IEEE congress on evolutionary computation (CEC) (pp. 1103-1110). IEEE.

Steinberg, L. (1961). The backboard wiring problem: A placement algorithm. Siam Review, 3(1), 37-50.

Taillard, É. (1991). Robust taboo search for the quadratic assignment problem. Parallel computing, 17(4-5), 443-455.

Tosun, U. (2015). On the performance of parallel hybrid algorithms for the solution of the quadratic assignment problem. Engineering applications of artificial intelligence, 39, 267-278.

Tsutsui, S., \& Fujimoto, N. (2009, July). Solving quadratic assignment problems by genetic algorithms with GPU computation: a case study. In Proceedings of the 11th Annual Conference Companion on Genetic and Evolutionary Computation Conference: Late Breaking Papers (pp. 2523-2530).

Xia, Y., \& Yuan, Y. X. (2006). A new linearization method for quadratic assignment problems. Optimisation Methods and Software, 21(5), 805-818.

Zhang, H., Beltran-Royo, C., \& Ma, L. (2013). Solving the quadratic assignment problem by means of general purpose mixed integer linear programming solvers. Annals of Operations Research, 207(1), 261-278.

Zhao, Q., Karisch, S. E., Rendl, F., \& Wolkowicz, H. (1998). Semidefinite programming relaxations for the quadratic assignment problem. Journal of Combinatorial Optimization, 2(1), 71-109. 


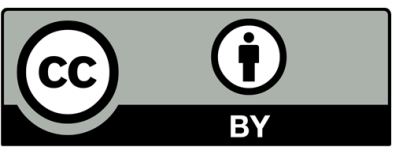

(C) 2022 by the authors; licensee Growing Science, Canada. This is an open access article distributed under the terms and conditions of the Creative Commons Attribution (CCBY) license (http://creativecommons.org/licenses/by/4.0/). 